Serum 6.-Rabbit Immunised with Strain 6, obtained fróm Boulogne.

\begin{tabular}{|c|c|c|c|c|c|c|c|c|c|}
\hline \multirow{2}{*}{ 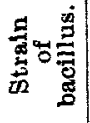 } & \multicolumn{9}{|c|}{ Dilution of serum. } \\
\hline & $1 / 50$ & $1 / 100$ & $1 / 200$ & $1 / 400$ & $1 / 800$ & $1 / 1600$ & $1 / 3200$ & $1 / 6400$ & $1 / 12800$ \\
\hline 1 & 0 & 0 & 0 & 0 & 0 & 0 & 0 & 0 & 0 \\
\hline 2 & $\dagger$ & 0 & 0 & 0 & 0 & 0 & 0 & 0 & 0 \\
\hline 3 & 0 & 0 & 0 & 0 & 0 & 0 & 0 & 0 & 0 \\
\hline 4 & $\dot{\dagger}$ & $\dagger$ & $\dagger \dagger$ & 0 & 0 & 0 & 0 & 0 & 0 \\
\hline 5 & 0 & 0 & 0 & 0 & 0 & 0 & 0 & 0 & 0 \\
\hline 6 & titt & $\dot{i t t i ́}$ & ††† & $T+\dagger$ & †ิT十 & ††† & t+ti & $t+t \dagger$ & tott \\
\hline $7-9$ & 0 & 0 & 0 & 0 & 0 & 0 & 0 & 0 & 0 \\
\hline
\end{tabular}

Serum 7.-Rabbit immunised with Strain 7 , obtained from Boulogne.

\begin{tabular}{|c|c|c|c|c|c|c|c|c|c|c|c|c|}
\hline \multirow{2}{*}{ 造 } & \multicolumn{6}{|c|}{$\begin{array}{l}\text { Dilution of serum. } \\
\text { Control }=0\end{array}$} & \multicolumn{6}{|c|}{$\begin{array}{l}\text { Dilution of serum. } \\
\text { Control }=0\end{array}$} \\
\hline & $\frac{8}{2}$ & $\underset{-1}{\stackrel{8}{8}}$ & 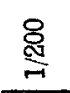 & $\underset{9}{8}$ & $\frac{8}{8}$ & 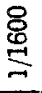 & $\stackrel{8}{2}$ & $\underset{ }{\stackrel{8}{8}}$ & $\underset{\mathrm{O}}{\stackrel{8}{-1}}$ & 兽 & 总 & 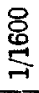 \\
\hline 1 & 0 & 0 & 0 & 0 & 0 & 0 & tith & 0 & 0 & 0 & 0 & 0 \\
\hline 2 & 0 & 0 & 0 & 0 & 0 & 0 & titt & †t十斤 & $t+t+$ & $\mathrm{itt+}$ & titt & 0 \\
\hline 3 & 0 & 0 & 0 & 0 & 0 & 0 & itt & $\dot{\dagger} \dagger$ & 0 & 0 & 0 & 0 \\
\hline 4 & 0 & 0 & 0 & 0 & 0 & 0 & $\dagger \dagger \dagger$ & $\dagger \dagger$. & 0 & 0 & 0 & 0 \\
\hline 5 & 0 & 0 & 0 & 0 & 0 & 0 & tit & †计 & 0 & 0 & 0 & 0 \\
\hline 6 & 0 & 0 & 0 & 0 & 0 & 0 & $\dagger+\hat{\imath}$ & †t† & 0 & 0 & 0 & 0 \\
\hline 7 & $\mathrm{ttth}$ & †t† & ttt† & ttt† & $\dagger \dagger$ & 0 & $\dagger \dagger$ & t†tt & titt & $t+$ & 0 & 0 \\
\hline 8 & $\dagger$ & $\dagger$ & 0 & 0 & 0 & 0 & itit & ttit & t†t & ItTT & Ifț & 0 \\
\hline 9 & 0 & 0 & 0 & 0 & 0 & 0 & & & & & & \\
\hline
\end{tabular}

Conclusion.

These results show clearly that the different strains of $B$. Pfeiffer are very different in their agglutination reactions, and as there was so much difference between them it seemed quite unprofitable to engage in a more extended series of tests. In most of the cases the agglutinins formed are either quite specific to the particular strain injected, or they are very nearly so, and in every case but one agglutination was observed in a much higher dilution with the homologous strain than with any of the others. The one exception (8) agglutinated one of the heterologous strains to the same titre as the homologous strain, and it agglutinated all the other strains tested to a lesser extent.

In marked contrast to this is the case of Serum 3, which agglutinated the homologous strain to a dilution of $1 / 1600$, but gave no agglutination with any of the others even in a dilution of 1 in 50 .

While this work was being done a communication was received through the Medical Research Committee from Dr. Park, of New York, who had been working on the same lines. Dr. Park obtained results similar to those set forth above, showing that almost every strain as regards agglutination is a law unto itself. He had had the opportunity, however, of testing the reactions of Pfeiffer's bacillus obtained from different members of a small community who had obviously been infected with influenza from a common source. In this small community the different strains of Pfeiffer's bacillus were entirely different as regards the agglutination test.

These results indicate that the $B$. influenze of Pfeiffer is really an associated group of bacteria without the marked unity in its immunity reactions as is seen in other epidemiccansing bacteria, such as typhoid and cholera.

So far as our present knowledge of epidemic bacteria goes, the facts set out above, agreeing as they do with Dr. Park's results in New York, are a strong indication that Pfeiffer's bacillus is not the primary cause of influenza, but that the primary infective agent awakens the activity of organisms which have been leading a more or less saprophytic existence in the respiratory tract.

These results are also of importance in connexion with the preparation of stock vaccines of Pfeiffer's bacillus. The results obtained during the last epidemic have shown that such a vaccine is valuable both for prophylactic and therapeutic purposes, and the experiments cited above indicate that such a stock vaccine should consist of a very large number of strains. It might be that in the vaccine there were a number of strains similar to Strain 8 , which stimulated the production of antibodies to many other strains; but it seems more likely that most of the strains would be less complete antigens, so that in the present state of our knowledge it would be desirable to incorporate many strains of Pfeiffer's bacillus in the vaccine to make it widely polyvalent.

In conclusion, we wish to thank Colonel S. L. Cummins, who, while Adviser in Pathology to the British Army in France, was the means of our being supplied with strains of Pfeiffer's bacillus from different areas.

\section{RUPTURE OF INTESTINE WITHOUT EXTERNAL INJURY.}

BY LLEWELLYN LINDOP, M.R.C.S. ENG., L.R.C.P. LOND., SURGEON COMMANDER, R.N.; SENIOR MEDICAL OFFICER, SHOTLEY.

IN Mr. Gerald Stanley's and Mr. W. H. Battle's very interesting papers on tranmatic rupture of intestine without external wound in your issues of July 19th and Oct. 25th I see, in discussing the cause of the rupture, that $\mathrm{Mr}$. Stanley says (p. 727):-

"If the abdomen is struck above the umbilicus the force is transmitted to the first few feet of the jejunum. The mobility of this coil allows it to be pushed till it is checked ultimately by the vertebral column, and between the latter and the moving body it is crushed and ruptured."

I am inclined to think there are two other factors: (1) Hydrostatic pneumatic effect ; (2) kink.

The small intestine contains fluid and gas, sometimes more, sometimes less. Any sudden violent compression applied to the abdomen tends to force fluid and gas out of the coils in its way. This force, acting on fluid and gas in a tube, acts equally and in all directions, and at first tends to balloon the tube. If the ends are unobstructed the contents are forced, aided by the elastic recoil of the tube, in the direction of its length--path of least resistance. This goes on until contents, or pressure only if tube full, slams up against the kink where the resistance is absolute. The pressure, communicated in the case of a full tube, or actual movement of contents in one collapsed, accentuates the kink. The tube balloons and ruptures at its unsupported margin. A complete transverse rupture occurs in a tube full of fluid to which sudden pressure is applied. It slams against the kink (duodeno-jejunal flexure), as in the case described by Mr. Stanley. It is the full tube which causes the rupture. It will be arguea that fluid and yas should go down as well as up. So they do. Some goes up and some goes down. But there is no kink below.

In rupture of intestine a small rent indicates gas and a large one fluid contents.' Gas is compressible, but fluid, for the purposes of this tube, absolutely incompressible. In the case of a full tube there is no actual movement of contents, only transmission of force. The duodenum ruptures above the duodeno-jejunal kink, and the ileum below. Any coil of small intestine temporarily caught distended with gas and some fluid, and from this cause having a slight twist, supplies its own kink. It is already ballooned, and if sufficient force is applied it ruptures at its unsupported margin. Rapture is more serious in the upper part of this tube, as there is more fluid and less gas. Gas tends to remain in the stomach and not pass on. The effects of sudden pressure are much more smashing on a tube distended with fluid than one distended with gas. In the lower part of the intestine any distension is caused by gas and effects of sudden pressures are less noticeable, and there are no kinks, except at the ileo-cral junction.

\section{Intestinal Gas and Fluid Pressure in other Abdominal Conditions.}

Duodenal and gastrie uleer.-I think the cause of the ulcer is not an erosion in the first place, but is an expression of damage to mucous membrane due to constant ballooning by plus pressure gas and fluid. The pressure acts at the pyloric end of the stomach and duodenum. If there is any disturbance with the mechanism at the pyloric sphincter, such as may be shown on the $X$ ray screen by delay after an opaque meal, the pylorus tends to balloon and is most unsupported on its anterior surface. Constant stretching 
causes a wound of the mucous membrane with subsequent irritant action of acid contents and bacterial infection due frequently to pyorrhoea. The ulcer is prevented from healing by the constant stretching, and when rupture takes place it needs a very little plus pressure to do it. An ulcer in the stomach is subjected to gas pressure. An ulcer in the duodenum is subjected to fluid pressure, hence the urgency of the symptoms of the rupture. A chronic dyspepsia, bad teeth, and constipation all cause gas.

Hernia.-I wondered for some time what force took the small intestine through the neck of a hernial sac. The force is : (1) Increased pressure inside the abdomen-dyspepsia and constipation, causing gas; (2) compression of abdominal cavity by muscles-strain, lifting weights, and application of force. Both forces usually act-effort causes attempted diminution in size of cavity containing gas. A small knuckle of intestine containing gas balloons through a hernial aperture and more gas passing through tends to distend it. Its margin is temporarily unsupported. The peritoneal surface of the intestine in health is smooth, shining, and slippery. The resistance due to ballooning of the engaged knuckle is obviously greater than friction to be overcome in bodily moving the tube.

A Richter's hernia is an example in which the force acts and balloons the unsupported margin, as it cannot move the tube owing to (1) adhesions, (2) undilatability of neck of sac owing to strong surrounding structure: e.g., femoralGimbernat's and Poupart's ligaments ; obturator-bone and thyroid membrane. The mechanism of reduction is to squeeze out the contents and then the gut follows-hence the familiar gurgle. Once gas is squeezed past the neck of the sac it pulls the gut back. Many hernias reduce themselves on lying down, as pressure in the abdomen is normally negative with slight positive variations.

Strangulated hernia.--The condition of the gut found at operation is not so much due to strangulation of the vessels as to ballooning and thinning of the intestine owing to gas pressure. There is an absolute kink at each end and gases are being formed by bacteria, \&c. This seems fairly obvious as the intestinal tube can be clamped during operation, its blood-supply absolutely cut off, with no urgent symptoms. The urgency of the symptoms is due to a kink at the upper end of the hernia affecting the tube above the hernia.

Hernia operations.--Plus pressure in the abdomen, either caused by strain, gas, or a combination of the two, tends to balloon intestine through any aperture. Operations should close the aperture, leaving a smooth well-supported surface with no pockets. If any pockets are left, the gas in the gut tends to dilate them as the pressure acts equally in all directions. In recurrent operations I rather think more care should be taken to keep abdominal pressure negative.

Appendicitis. - Put in a word, the mechanism here is : appendix-short mesentery, kink ; cæcum-fluid and gas pressure. Bad teeth lead to a preference for carbohydrate diet, which is easier to chew. Bad teeth and diet lead to dyspepsia and constipation, which means gas production. In the cæcum there is increased pressure, and cæcum tends to balloon. This does not do much harm to the cæcum. It is quite a different matter for the appendix to which the pressure is transmitted. It cannot balloon much and tends to rupture at the kink. If peritonitis occurs as a result of perforation of the appendix, it is caused by a sudden great increase of pressure. If an abscess occurs as a result of perforation it is due to chronic small increase of pressure. Enemas in "observation" appendix cases may do good or do harm. If they relieve the pressure by removing contents of the colon and gas the pressure falls and the appendix will not rupture immediately. If the usual measures are taken (starvation, \&c.) the case gets well without operation. On the other hand, if they remove some of the contents of the colon but no gas, they may actually increase the pressure and cause the rupture.

Constipation. - Gas caused by bacteria, \&c., balloons the tube, as colon is unsupported in the correct position except at kinks. This condition becomes permanent as intestinal muscle loses tone. The ballooning accentuates the kinks and so actually obstructs the passage of the contents. All secondary effects are due to invasion of the mucous membrane of the tube with orgarisms, \&c.

$$
\text { Gas Pressure. }
$$

In conclusion, I may add I have purposely laid stress on gas pressure in the abdomen as an exciting cause of acute and chronic abdominal conditions. Ererybody is agreed as to gaseous distension after the condition is produced, but I do not think its effect as a cause of abdominal conditions has been sufficiently recognised, except perhaps in intussusception and volvulus. Sir Arbuthnot Lane's operation is designed to relieve the patient of a colon damaged by gas and infected by various organisms. The absence of the intestinal contents means no gas, as there is nothing for the organisms to grow on.

Gas pressure is the origin of intestinal movements. It excites peristalsis in a normal tube and if increased or often applied in a tube with kinks, causes ballooning and pouch. ing. Organisms present cause gas. Most purgatives act by increasing gas formation and excite increased peristalsis, hence their gradual failure ; the tube ceases to react to the pressure and dilates. It seems very unwise often to subiect a tube, with as many kinks as the colon, to increased pressure. When this has been done, usually by the patient having himself used purgatives wholesale, the advisability of short-circuiting, to relieve him of the secondary toxic effects, has to be considered. Mr. Fletcher's method of eating seems to reduce the gas formation to a minimum. People who bolt their food may actually swallow gas with each bolus; otherwise the bolus would stick, but wrapped in ac air cushion it passes safely on.

\section{Clinital atotes:}

MEDICAL, 'SURGICAL, OBSTETRICAL, AND THERAPEUTICAL.

$$
\text { A CASE OF }
$$

GENERALISED TUBERCLLOSIS PRESENTING DIFFICULTIES IN DIAGNOSIS.

By F. J. NatTrass, M.B., B.S. DurH., M.R.C.S. Elio., L.R.C.P. LOND.,

CAPTAIN, R.A.M.C. (T.), SPECIAL MILITARY SURGICAL HOSPITAI [NEWCASTLE-UPON-TYNE.

THE following case of obscure illness is of interest in illustrating the difficulty in diagnosis of deep-seated tuberculous infection, and the value of $\mathrm{X}$ ray examination of the chest in such cases.

Pte. J. R. R., aged 18 years, with nine months' service. was admitted to the 1st Northern General Hospital, Newcastle upon-Tyne, April 7th, 1919. The history was that in the previous month he spent a week in hospital with influenza a week after discharge from hospital he began to have pain and swelling in the right hand and pain in both hips. On admission temperature was $103^{\circ} \mathrm{F}$., pulse 110 , respirations 24 , and the case was diagnosed as acute rheumatism. Sodium salicylate was administered, but the temperature remained high, varying in the evenings from $100.5^{\circ}$ to $102^{\circ}$, with morning remissions of about one degree. This was the condition when the case was transferred to me on May 6th, on the demobilisation of the 1st Northern General Hospital. The patient was pale and sweating, had lost mach weight, but was not markedly emaciated ; in the neighbourhood of the right.carpo-metacarpal joints there was a painful fluctuating swelling, which was not red and showed no sign of pointing. The pain in the hips, which had never been gevere, was now only glight. $X$ ray photographs of the hand showed slight blurring of the joint surfaces between the carpal bones and the three inner metacarpals ; X ray photographs of the hips showed no abnormality. There was no sign of endocarditis, no discoverable abnormality in the abdomen, no cough, and the only physical sign of disease other than the swelling of the hand was a localised dry pleuritic rub at the base of the right lung. Within four or five days this rub disappeared, and was replaced by an occasional crepitation at the bases of both lungs posteriorly; these crepitations, however, also disappeared in a few days, and after this no physical signs of disease could be found in the chest, on repeated examination, until within ten days of death, which took place on July 4th, 1919.

As the diagnosis of acute rheumatism appeared to be definitely negatived careful search was made for other forms of infection. Specimens of urine, passed in the early morning. and the centrifugalised deposit of 24 hour's collection, were examined by Dr. P. C. W. Laws for gonococci with negative results. On the supposition of a 